Recepción: 26 / 04 / 2018

Aceptación: 13 / 06 / 2018

(c) 1 (1)(2)

Ciencias de la educación

Publicación: 01 / 08 / 2018

Artículo de investigación

\title{
Impacto de las redes sociales en el contexto educativo
}

\author{
Impact of social networks in the educational context
}

Impacto das redes sociais no contexto educativo

\author{
Cristina A. Delgado-Rivera I \\ cristina.delgado@unae.edu.ec \\ Alicia G. Paredes-Benavides II \\ agparedes@unae.edu.ec \\ Jacqueline E. Plaza-Loyola ${ }^{\text {III }}$ \\ jeplaza@unae.edu.ec \\ Gloria L. Gualpa-Marca IV \\ glgualpa@unae.edu.ec
}

Correspondencia: cristina.delgado@unae.edu.ec

\footnotetext{
I Magíster en Gerencia de Salud para el Desarrollo Local, Psicóloga Clínica, Docente de la Universidad Nacional de Educación, Manabí, Ecuador.

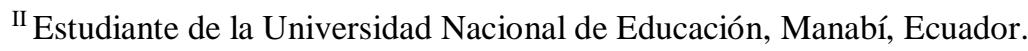

III Estudiante de la Universidad Nacional de Educación, Manabí, Ecuador.

IV Estudiante de la Universidad Nacional de Educación, Manabí, Ecuador.
} 


\section{Resumen}

Este proyecto de investigación consiste en analizar críticamente, el Impacto de las Redes Sociales en el contexto educativo. La comunicación y la expresión libre del ser humano, como un ser sociable, se dan a través de la participación y cooperación en los distintos contextos de la vida cotidiana. Las redes sociales son uno de los espacios en el que los sujetos interactúan. Los principales instrumentos utilizados para la recolección de datos fueron: observación participante y encuestas, los mismos que fueron aplicados a los estudiantes de primero de bachillerato pertenecientes a la Unidad Educativa "Herlinda Toral". Por medio de estos instrumentos conocemos que, las Redes Sociales más utilizadas por los estudiantes son: Facebook en un $33.33 \%$ mientras que los datos obtenidos del INEC mantiene a esta Red Social con un 97\%, Whatsapp con un $26.66 \%$ contrastando con los datos obtenidos del INEC con un $90 \%$ e Instagram con un 20\% y $65 \%$ del INEC, teniendo como principal uso "El Chat" intrapersonal. E1 uso negativo puede ocasionar un bajo rendimiento académico y destruir relaciones afectivas, sin embargo, su uso adecuado crea espacios de aprendizaje activos, en los cuales se estimula el pensamiento crítico.

Palabras clave: redes sociales; contextos; familiar; educativo; comunitario.

\section{Abstract}

This research project consists in critically analyzing the Impact of Social Networks in the educational context. The communication and the free expression of the human being, as a sociable being, are given through participation and cooperation in the different contexts of daily life. Social networks are one of the spaces in which subjects interact. The main instruments used for the data collection were: participant observation and surveys, which were applied to the students of the first year of high school belonging to the Educational Unit "Herlinda Toral". Through these instruments we know that the most used Social Networks by students are: Facebook in $33.33 \%$ while the data obtained from INEC keeps this Social Network with 97\%, Whatsapp with $26.66 \%$ contrasting with the data obtained of the INEC with $90 \%$ and Instagram with $20 \%$ and $65 \%$ of the INEC, having as main use "The Chat" intrapersonal. Negative use can cause low academic performance and destroy emotional relationships, however, its proper use creates active learning spaces, in which critical thinking is stimulated. 
Key words: social networks; contexts; family; educational; community.

\section{Resumo}

Este projeto de pesquisa consiste em analisar criticamente o impacto das redes sociais no contexto educacional. A comunicação e a livre expressão do ser humano, enquanto ser sociável, são dadas através da participação e cooperação nos diferentes contextos da vida cotidiana. As redes sociais são um dos espaços em que os sujeitos interagem. Os principais instrumentos utilizados para a coleta de dados foram: observação participante e pesquisas, o mesmo que foram aplicados aos calouros do ensino médio pertencentes à Unidade Educacional "Herlinda Toral". Através destes instrumentos sabemos que as redes sociais mais utilizadas pelos estudantes são: Facebook por 33,33\%, enquanto os dados do INEC mantido a esta rede social com 97\%, Whatsapp com 26,66\%, em contraste com os dados obtidos do INEC com $90 \%$ e Instagram com $20 \%$ e $65 \%$ do INEC, tendo como principal uso o "The Chat" intrapessoal. O uso negativo pode causar baixo desempenho acadêmico e destruir relacionamentos emocionais, entretanto, seu uso adequado cria espaços de aprendizagem ativos, nos quais o pensamento crítico é estimulado.

Palavras chave: redes sociais; contextos; familia educativo; comunidade.

\section{Introducción}

El presente estudio de carácter social-educativo se centra en el tema Impacto de la Redes Sociales en el Contexto Educativo, con el fin de conocer los impactos que provocan estas herramientas de comunicación en los estudiantes.

Debido a la inmensa cantidad de información digital se han producido cambios en la sociedad (Scroder, 2018) ya que, en los últimos años se ha generado una revolución tecnológica la misma que tiene sus orígenes en 1969, con la creación del internet (Díaz \& Arab, 2015).

El uso de las redes sociales ayuda a la comunidad de educadores para la cultura científica, intercambiando información, abriendo paso al debate, generando planes y sugerencias vinculadas en el papel de las Redes Sociales y las colectividades; ocupando un lugar principal en el contexto político y en las tácticas de educación, formación y el desarrollo profesional. Sin embargo, modifica las formas de vida, renovando las prácticas y creando un nuevo léxico. Esto se produce 
a un ritmo vertiginoso, generando ambigüedad y desinformación relacionado con la identidad y privacidad (Monterosa Carvajal, 2017).

El Instituto Nacional de Estadística y Censos (INEC) según la encuesta de Empleo Desempleo y Subempleo publicó que en el año 2016; 4,2 millones de personas usaron Redes Sociales, esta cifra tiene relación con el número de usuario con Smartphones (teléfonos inteligentes). Los Smartphones han creado un contexto diferente para que los seres humanos puedan comunicarse, crear conocimientos y compartirlos ya que cambian los modos de crear y transferir el conocimiento buscando desarrollar las nuevas competencias necesarias para desenvolverse en la sociedad del conocimiento y agilizan el flujo constante de información (Hampton, y otros, 2016).

El INEC constató cuales son la Redes Sociales más utilizadas por los jóvenes, ubicándose en el siguiente orden: Facebook con un 97\%, WhatsApp con un 90\%, Instagram con un 65\%, Twitter con un $49 \%$.

En la actualidad, los adolescentes pasan un promedio de siete horas y media interactuando en las Redes Sociales que nos indican la necesidad de introducirse en el campo de investigación en cuanto a hábitos y consumo de niños y jóvenes en las redes sociales (Judge, Floyd, \& Jeffs, 2015) (Ahern, Feller, \& Jeffs, 2015) comprendiendo que es recomendable utilizarlas de dos a tres horas diarias, siendo el sector urbano el área en la cual se emplean con mayor frecuencia $(81,9 \%)$.

En cuanto al sexo, se comprobó que los hombres utilizan las Redes Sociales en un 49,9\% y las mujeres en un 50,1\%, en las edades que más llaman la atención es entre los 12-17 años, comprendiendo que, los estudiantes que cursan la secundaria son los más atraídos por las mismas (García Guerrero, 2016).

En la actualidad, las Redes Sociales crean un gran interés, especialmente en jóvenes, debido a su constante actualización e incremento, su principal atracción es el poder comunicarse con personas de todo el mundo (Martos, 2010), por este motivo se investiga qué tan importante son estas herramientas de comunicación en estos determinados individuos. Además, han contribuido mucho al desarrollo de las sociedades contemporáneas conformando el nuevo modelo de las personas (del Dujo García \& Muñoz, 2015). 


\section{Metodología}

El presente estudio es descriptivo, debido a que se emplean métodos de aproximación cualitativa y cuantitativa. Además, se utilizaron la observación participante y encuestas a un total de treinta y ocho estudiantes de una Unidad Educativa de la Ciudad de Cuenca.

Cuantitativa: de esta manera conoceríamos el porcentaje de estudiantes que hacen uso de las Redes Sociales y para qué fines utilizan las mismas.

Cualitativa: sería detectar los comportamientos y gustos que tienen los estudiantes hacia las diferentes Redes Sociales.

\section{Instrumentos}

\section{Observación Participante}

Es la descripción de grupos sociales y escenas culturales, mediante la vivencia de las experiencias de las personas implicadas en un grupo donde el observador se hace partícipe de los fenómenos que observa (Calvo Pérez, Técnicas e instrumentos de diagnóstico en educación, 2007).

Cuando realizamos las prácticas pre-profesionales tuvimos la oportunidad de realizar observaciones directas, considerando a éste como primer instrumento de recolección de datos. A través de este método observamos el empleo de las Redes Sociales por parte de los estudiantes mediante herramientas tecnológicas.

\section{Encuestas}

Conjunto de operaciones necesarias para descubrir la verdad sobre un hecho, no es un método específico, pero es una técnica de gran utilidad en las ciencias de la conducta (Calvo Pérez, 2007, pág. 95).

Para este proyecto se realizaron encuestas a los estudiantes de la Unidad Educativa "Herlinda Toral", el curso encuestado fue el siguiente:

1ero "A" Comercio Exterior 38 estudiantes.

Los datos obtenidos fueron analizados mediante programas informáticos como Excel y Nvivo, con el fin de recolectar información que respalden nuestros objetivos. 


\section{Presentación de los resultados}

En este punto se presenta los resultados finales de las preguntas que se plantearon en las encuestas, las cuales se realizaron a treinta y ocho estudiantes. De esta manera, se conoce las preferencias de las Redes Sociales y los fines con los cuales utilizan las mismas.

Gráfico 1. Representa las preferencias que tienen los estudiantes en cuanto a Redes Sociales. Se constata así, que más del 30\% utilizan Facebook como su principal herramienta de comunicación.

Facebook

Whatsapp

Instagram

Snapchat

Twitter
$33.33 \%$

$26.67 \%$

$20.00 \%$

$13.33 \%$

$6.67 \%$

\section{Redes Sociales más utilizadas por los estudiantes}

33.33

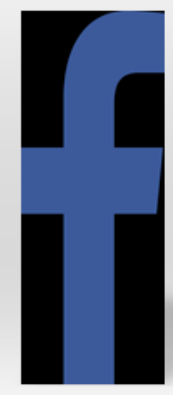

Facebook Whatsapp

26.67

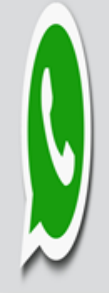

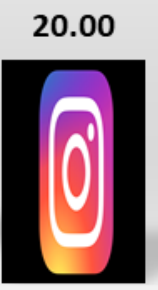

13.33

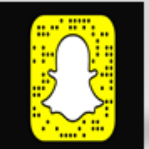

6.67

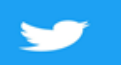

Instagram

Snapchat

Twitter

Fuente: elaboración propia (Gualpa, Paredes, Plaza) 2018.

Datos recopilados de las encuestas aplicadas. 
Gráfico 3. Se conoce qué tan elevado es el porcentaje de horas que los estudiantes dedican a las redes sociales.

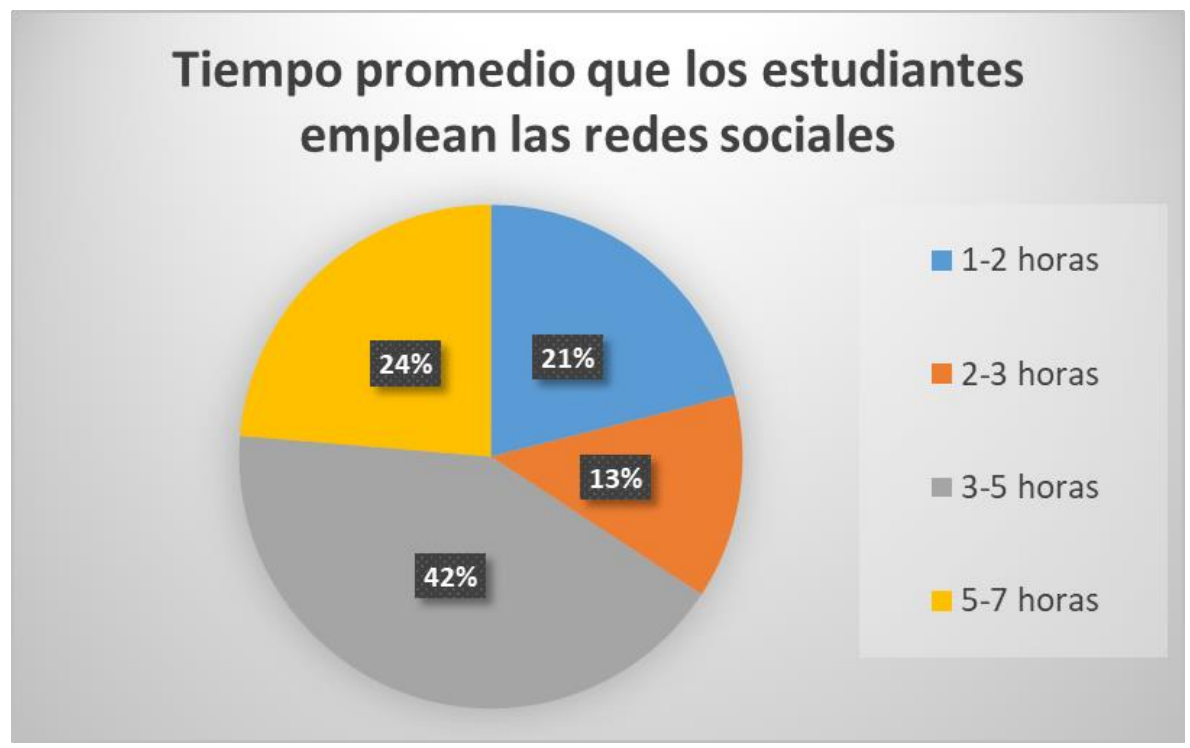

Fuente: elaboración propia (Gualpa, Paredes, Plaza) 2018.

Datos recopilados de las encuestas aplicadas.

Gráfico 6. Se comprende el motivo por el cual los estudiantes utilizan las redes sociales, verificando que la mayoría utiliza estas herramientas para chatear en un $65,79 \%$.

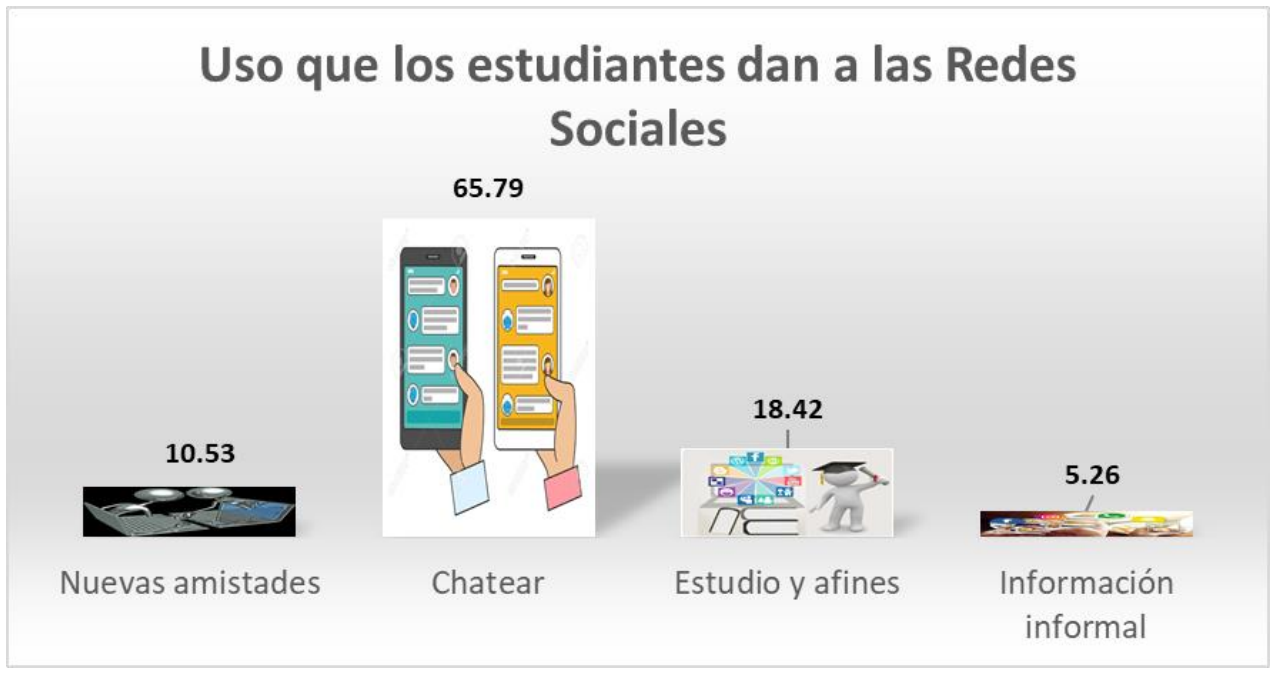

Fuente: elaboración propia (Gualpa, Paredes, Plaza) 2018.

Datos recopilados de las encuestas aplicadas. 
A continuación, se presentan los datos cualitativos obtenidos a través de las preguntas abiertas.

Gráfico 7. De acuerdo a la información adquirida se comprueba que, las Redes Sociales influyen en las distintas relaciones interpersonales, de manera positiva: existe la comunicación sin límites con personas de cualquier parte del mundo; y negativa: se crean amistades falsas y superficiales, alejándose de las personas cercanas y confiables con quienes convive el estudiante.

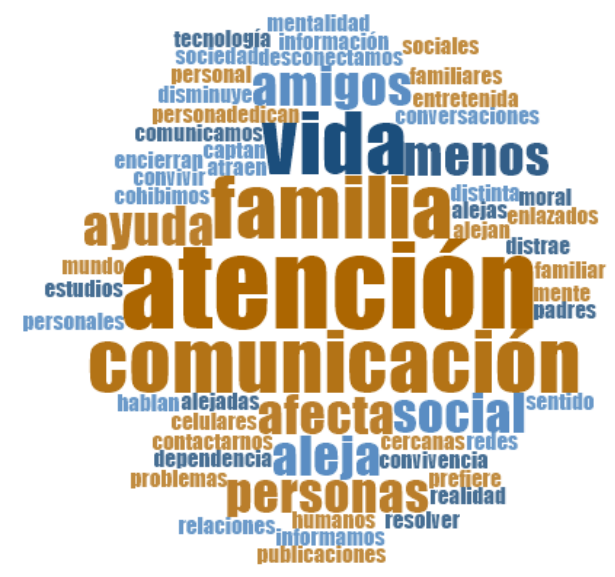

Fuente: elaboración propia (Gualpa, Paredes, Plaza) 2018.

Datos recopilados de las encuestas aplicadas.

Gráfico 8. En relación con la información analizada, para el estudiante, las Redes Sociales afectan en su rendimiento académico, de manera positiva: los estudiantes tienen acceso a una variedad de información que favorece a su desempeño académico; y negativa: existe distracción al momento de estudiar o realizar tareas ya que, la mayoría del tiempo chatean.



Fuente: elaboración propia (Gualpa, Paredes, Plaza) 2018.

Datos recopilados de las encuestas aplicadas 


\section{Discusión}

En este punto, se contrasta la información adquirida a través de los instrumentos de recolección de datos anteriormente mencionados, es decir, la realidad del uso de las Redes Sociales con lo que se estipula en los diversos documentos que se indagaron para realizar la sistematización teórica.

De acuerdo con la investigación realizada del INEC a nivel nacional se reconoce a Facebook en un 97\% como la Red Social más usada por adolescentes, sin embargo, el objeto de estudio lo acepta con un 33.33\%, lo mismo sucede con WhatsApp como segunda opción es aceptada con un $90 \%$ mientras que la muestra de la población la acepta con un $26.66 \%$ e Instagram como su tercera opción en la cual, según el INEC es de un 65\% y la muestra lo acepta con un 20\%. De la misma manera, el Smartphone tanto en la encuesta como en la investigación se mantiene en el primer puesto. Los factores que influyen en la variación de porcentajes entre la muestra nacional y el muestreo según (Manzano, 2009-2013) dependen de: el tamaño de la población, variación en la población y el error de la precisión, cabe recalcar que, este es un tema de continua investigación, y es recomendable continuar analizando las diferencias en los resultados encontrados.

Cuando hablamos acerca de las nuevas tecnologías, específicamente las Redes Sociales, surge una duda acerca del tiempo considerable que el estudiante le debería dedicar a las mismas, el INEC recomienda utilizarlas de 2 a 3 horas diarias sabiendo que el 13\% lo cumple, también, el $21 \%$ las utilizan adecuadamente de 1 a 2 horas, sin embargo, el $42 \%$ de los estudiantes las utilizan de 3 a 5 horas diarias y el $24 \%$ de 5 a 7 horas diarias, esta diferencia con la media nacional puede deberse a distintos factores, que se sugiere sean investigados en otro estudio sobre este tema.

La información obtenida con respecto al impacto que tienen las Redes Sociales en los distintos contextos: familiar, comunitario y educativo contrastado con los datos obtenidos a través de las encuestas, podemos decir lo siguiente:

Educativo: Según (Judge, Floyd, \& Jeffs, 2015), en las Redes Sociales se crean espacios de aprendizaje informales, los cuales, se convierten en factores importantes para el estudio, de la misma manera, (Díaz \& Arab, 2015) explica el estímulo en zonas cerebrales que ayudan a 
alcanzar las metas planteadas. Sin embargo, manifiesta que, un mal uso de estas herramientas produce una disminución del rendimiento académico, abandono escolar, un déficit de atención debido a que, según (Berkin, 2015) las utilizan más con fines de entretenimiento y no con fines educativos ocasionando fallas en el proceso de aprendizaje.

Familiar: según (Hampton, y otros, 2016) (Han, 2014) manifiestan que las Redes Sociales inciden en la forma de conocer el mundo y expresar las ideas propias, por este motivo, los padres deben ayudar a fortalecer su personalidad ya que, para otros autores, la falta de apoyo ocasiona depresión, hiperactividad, insomnio, cambios de estados de ánimo y crea relaciones inestables (Muller, Janikian, Wolfling, Beutel, \& Tzavara, 2014) (Yao, Chen, Wang, JT, \& Zue, 2014) (Dalbudak, Evren, Aldemir, \& Evren, 2014). Aunque, (Miranda, Morales, Castellanos, \& León, 2008) valorizan que, estos espacios sirven para un intercambio activo entre sus integrantes, posibilitando y potenciando recursos que se poseen con el fin de enriquecer múltiples relaciones afectivas.

Comunitario: para (Díaz \& Arab, 2015) en este contexto los adolescentes generan estrategias adaptativas y no adaptables que favorezcan al desarrollo de su identidad integral. Buscando sentimientos de pertenencia y acceso a la información creando una autoestima colectiva. Estas herramientas tecnológicas generan y exploran aspectos de la identidad las cuales pueden ser experiencias interpersonales e intrapersonales enriquecedoras o destructivas según el uso que se les den (Argentina, Ministerio de Educación, 2010).

(Hampton, y otros, 2016) (Han, 2014) manifiestan que las Redes Sociales permiten compartir información e intereses comunes con otros usuarios tal y como el 65,79\% de los estudiantes lo emplean con el fin de "chatear" o interactuar con sus diversos contactos mientras que, el 18,42\% de los estudiantes están conscientes que, estas herramientas pueden servir a la hora de reforzar conocimientos, como lo menciona (Monterosa Carvajal, 2017) quien está de acuerdo con el intercambio de información científica que abre paso al debate entre las distintas colectividades. 


\section{Conclusiones:}

Las redes sociales más utilizadas por los estudiantes encuestados son: Facebook, Whatsapp e Instagram verificando que el Smartphone es el dispositivo más empleado para ingresar a las mismas.

El motivo principal por el que los estudiantes utilizan las Redes Sociales es para mantenerse en contacto con otros usuarios, aunque, un cierto porcentaje las utilizan con fines educativos.

El mal uso de las Redes Sociales suele ocasionar un bajo rendimiento académico, destruir relaciones personales y familiares según la perspectiva del estudiante. Sin embargo, el uso adecuado de estas herramientas puede crear espacios de aprendizaje interactivos en los cuales, el estudiante puede reforzar lo que aprendió en clases.

Al realizar la investigación, se han encontrado diversas y grandes diferencias entre los porcentajes nacionales con los porcentajes del muestreo, por esta razón es recomendable hacer de estos temas un factor de estudio con el fin de descubrir cuáles son los motivos de estos resultados.

\section{Referencias Bibliografícas}

Ahern, L., Feller, K., \& Jeffs, T. (2015). Using mobile media devices and apps to promote young children's learning. En L. Heider, \& M. Jalongo, Young children and families in the information age, educating the young child (págs. 117-131). New York: Spring-ger.

Argentina, Ministerio de Educación. (2010).

Berkin, S. (2015). Comunicación y sociedad.

Calvo Pérez, C. (2007). Tecnicas e instrumentos de diagnóstico en educación. Málaga: ALJIBE.

Calvo Pérez, C. (2007). Técnicas e instrumentos de diagnóstico en educación. Málaga: ALJIBE.

Dalbudak, E., Evren, C., Aldemir, S., \& Evren, B. (2014). The severity of internet addiction risk and it relationship with the severity of borderline personality features, childhood traumas, dissociative experiences, depression and anxiety symptoms among Turkish university students . Pubmed.

del Dujo García, A., \& Muñoz, J. (2015). Medios de interacción social y procesos de (de-re) formación de ciudadanias. 
Díaz, A., \& Arab, E. (2015). Impacto de las redes sociales e internet en la adolescencia: aspectos positivos y negativos.

García Guerrero, J. (julio de 2016). INEC. Obtenido de La encuesta de condiciones de vida.: http://www.ecuadorencifras.gob.ec/documentos/web-inec/boletin/E-commerce.pdf

Hampton, K., Rainie, L., Lu, W., Dwyer, M., Shin, I., \& Purcell, K. (2016). Social Media and the spiral of silence. Pew Research Center.

Han, B. (2014). El enjambre . Barcelona: Herder.

Judge, S., Floyd, K., \& Jeffs, M. (2015). Children and their changing media environment: A European comparative study. London: Routledge.

Manzano, V. (2009-2013). El tamaño de la muestra.

Martos, E. (2010). Análisis sobre las nuevas formas de comunicación a través de las comunidades virtuales y sociales. En E. Martos. Valencia: gandia: Universidad de Valencia.

Miranda, C., Morales, M., Castellanos, M., \& León, A. (2008). Redes sociales de conocimiento tradicional: escencia, historia y trayectorias. Bogotá: Cyted.

Monterosa Carvajal, A. (2017). En A. Monterosa Carvajal, Administración de clases y gestión del conocimiento (págs. 2-7).

Muller, K., Janikian, M., Wolfling, K., Beutel, M., \& Tzavara, C. (2014). Regular gamin behavior and internet gamin disorder in European adolescents: results from a cross-national representative survey of prevalence, predictors, and psychopathologycal correlates. Epub ahead of print.

Murillo T, J. (2007). La entrevista.

Scroder, R. (2018). Towards a theory of digital media. Information communication and society. Yao, Y., Chen, P., Wang, L., JT, Z., \& Zue, G. (2014). Failure to utilice feedback causes decision-making deficits among internet gamers. Psychiatry ress. Pubmed. 